\title{
Metastatic alveolar soft part sarcoma masquerading as primary CNS tumor
}

\author{
Deepak K. Goel ${ }^{1}$, Priti P. Trivedi ${ }^{2, *}$, Shailee P. Mehta ${ }^{3}$, Paresh G. Mody ${ }^{4}$, Dipak D. Patel ${ }^{5}$ \\ ${ }^{1}$ Resident, ${ }^{2}$ Associate Professor, ${ }^{3}$ Fellow, ${ }^{4,5}$ Consultant Neurosurgeon, ${ }^{1-3}$ Dept. of Pathology, ${ }^{4,5}$ Dept. of Neuro-Oncology, Gujarat \\ Cancer Research Institute, Ahmedabad, Gujarat, India
}

*Corresponding Author:

Email: priti_patho@yahoo.co.in

\begin{abstract}
Alveolar soft part sarcoma (ASPS) is a very rare malignant, highly aggressive and profusely vascular soft tissue sarcoma of controversial histological origin accounting for less than $1 \%$ of the soft tissue sarcoma cases worldwide. Though it has an indolent clinical course, however, because of its propensity to recur and frequently metastasize, it usually has a fatal outcome. The knowledge of its microscopic features and TFE3 staining facilitate earlier diagnosis. Surgical resection helps in increasing the disease-free period, but the role of adjuvant chemotherapy and radiotherapy is disputed. After resection, it should be frequently monitored because of its malignant potential and dismal prognosis. We present a case report of twenty-year-old male patient whose entire clinical presentation was that of a primary brain malignancy with no symptoms anywhere else in the body but was diagnosed to be a case of alveolar soft part sarcoma of para lumbar region with brain and lung metastasis. To the best of our knowledge, it is an extremely uncommon presentation with only fourteen cases have been reported so far.
\end{abstract}

Keywords: Alveolar soft part sarcoma, brain metastasis, TFE3 nuclear positivity.

\section{Introduction}

Alveolar soft part sarcoma (ASPS) is a malignant, highly aggressive and profusely vascular rare soft tissue sarcoma (STS) of controversial histogenesis, accounting for less than $1.0 \%$ of all STS cases, worldwide. ${ }^{1}$ It gained its existence in the medical literature as a separate entity in the year 1952. The average age of diagnosis is between 20 to 30 years with females tend to be affected more commonly and little early than male. Unlike most sarcomas, ASPS after arising from extremities is known to frequently metastasize, primarily to the lungs (42\%), bones (19\%), brain $(15 \%)$ and lymph nodes $(7 \%){ }^{2}$ Radical resection to achieve its complete removal is accepted as the treatment of choice for both primary tumors and metastatic tumors. ${ }^{3}$ Although ASPS is considered to have an indolent clinical course, it is considered to be high grade, and eventually, ASPS is usually a fatal disease with a poor prognosis. Children have better overall survival rate compared with adults.

We report a unique case of a young adult male of twenty years whose entire initial clinical presentation at the time of admission was that of primary CNS malignancy but was diagnosed as metastasis of ASPS from the paralumbar region to brain and lungs. To the best of our knowledge, only fourteen cases of alveolar soft-part sarcoma metastasis to brain mimicking a primary brain malignancy with no symptom at the site of its origin or metastasis other than brain have been reported so far. ${ }^{4}$

\section{Case Report}

A twenty-year-old male was referred for posterior fossa space occupying lesion for further management. $\mathrm{He}$ presented with a headache, dizziness, and intractable vomiting which progressively compounded over time. He had no other known medical illnesses, no addiction or relevant family history. On examination, his Glasgow Coma Scale (GCS) was 15 out of 15 and he was conscious and well oriented to time, place, and person. No palpable lymphadenopathy or hepatosplenomegaly were noted. Cardiovascular, respiratory, gastrointestinal and urogenital system findings were unremarkable.

On magnetic resonance imaging (MRI) brain, a dense enhancing space-occupying lesion in the right cerebellar lobe was seen with mass effect suggesting primary malignancy.

The patient was operated as the right cerebellar space-occupying lesion was solitary. Craniotomy with complete excision of the tumor was done after confirmation of it being a malignant tumor on frozen section. Intra-operative findings were a pale-yellow vascularized tumor with a mix of solid-cystic-friable areas with a rubbery feel.

On histopathological examination grossly, it was a single grey-white soft to firm tissue piece measuring $3.5 \times 3.0 \times 2.0 \mathrm{~cm}$. On microscopic examination (Fig.1), it showed nests of cells arranged in the alveolar pattern with intermixed blood vessels. Individual tumor cells were large with abundant eosinophilic cytoplasm, eccentrically placed nucleus with prominent nucleoli. Scattered mitosis was evident. Provisional diagnosis considered was metastatic tumor with following differential diagnosis:

1. MiT family translocation renal cell carcinoma

2. Paraganglioma

3. Metastatic Alveolar soft part sarcoma or primary alveolar soft part sarcoma 


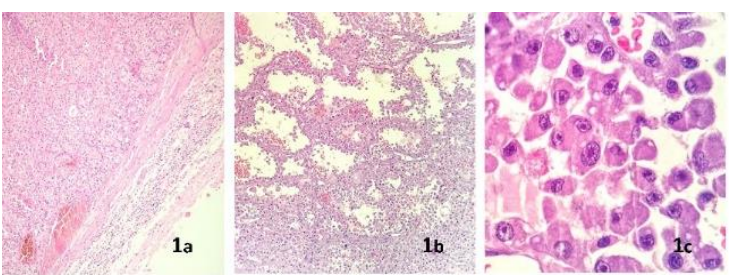

Fig. 1: (a) Tumor with normal brain parenchyma (H\&E, 40x), (b) alveolar pattern with vascular septa $(H \& E, 100 x)$ and (c) tumor cells with nuclear atypia, prominent nucleoli and abundant eosinophilic cytoplasm (H\&E, 1000x)

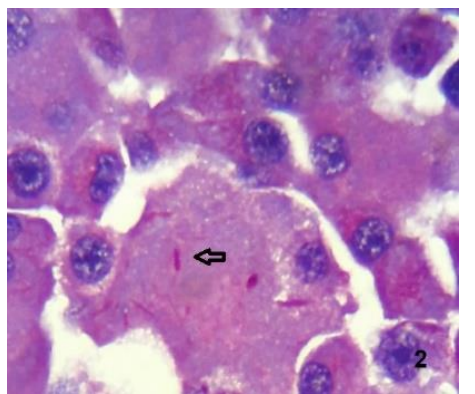

Fig.2: PAS positive diastase resistant needle-like crystals (black arrow)

Special stain: PAS and PAS diastase was given which demonstrated resistant needle like crystals (Fig.2).

Immunohistochemistry (IHC) was also performed. Tumor cells were positive for TFE-3 (Fig. 3) and negative for PAX8, CD10, CK7, AE1, EMA, TTF1, CD34, Synaptophysin, GFAP, CD117, OCT3/4, HMB 45, S 100 and Vimentin, thus confirming the diagnosis of alveolar soft part sarcoma (ASFS).

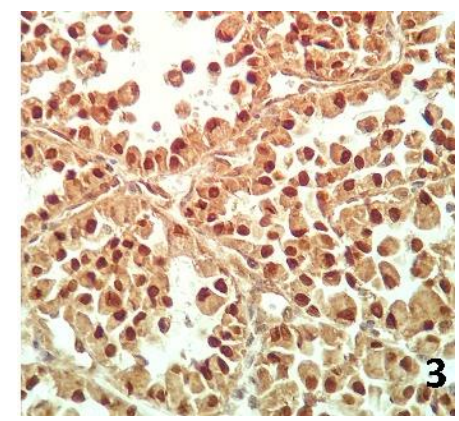

Fig. 3: TFE-3 with nuclear positivity

Positron emission tomography (PET) scan and Computed Tomography (CT) Scan was advised to diagnose the primary site.

CT scan revealed presence of multiple well-defined soft tissue opacities, the largest being $34 \times 29 \mathrm{~mm}$ in the right hilar region of lung suggestive of metastasis and in the PET scan increased Fluro-deoxy-glucose (FDG) uptake was noted in the right paramedian lumbar iliac fossa region indicating it to be the primary site. Also, metastasis in multiple mesenteric nodes and lungs was also noted.

The patient was started on chemotherapy.
1. Doxorubicin $20-25 \mathrm{mg} / \mathrm{m}^{2}$ IV push on days $1-3$ plus

2. Ifosfamide $2000-3000 \mathrm{mg} / \mathrm{m}^{2} \mathrm{IV}$ push bolus $3 \mathrm{~h}$ on days 1-3 repeated every three weeks for a total of total 6 cycles. Patient was asymptomatic in a follow up period of six months.

\section{Discussion}

Out of all the solid malignant tumors, Soft tissue sarcoma (STS) accounts for merely less than $1 \%$ and out of all the STS, ASPS accounts for less than $1 \%$ pointing towards the rarity of the disease. Christopherson et al. coined the term ASPS and first described its unique histological and cytological features in the year $1952 .{ }^{5}$ It has a slightly higher incidence in females of juvenile age group. ${ }^{6}$ In adults it mostly affects the soft tissues of the pelvic cavity and lower limbs; however, it is more commonly seen in the head and neck region in a younger age group.

Although, most researches have speculated it to be myogenic, origin of ASPS remains a matter of research. Smetana and Scott observed the pulmonary alveoluslike arrangements of the tumor cells in ASPS and described its pathological manifestations. It is primarily diagnosed based on the histological features of the tumour which are large polygonal cells arranged in an alveolar pattern with granular eosinophilic cytoplasm, vesicular nuclei, prominent nucleoli. ${ }^{5}$ They have PAS positive diastase resistant needle like crystals and show characteristic TFE3 nuclear positivity.

Paraganglioma, alveolar rhabdomyosarcoma, renal cell carcinoma, metastatic adrenal carcinoma, granular cell tumor and melanoma closely resembles ASPS and must be considered in its differential diagnosis.

ASPS is characterized by a specific unbalanced chromosomal translocation, $\operatorname{der}(17) \mathrm{t}(\mathrm{X}: 17)$ (p11: q25), resulting in the fusion of the TFE3 transcription factor gene (from Xp11) with alveolar soft part sarcoma critical region 1 (ASPSCR1) which is also known as alveolar soft part sarcoma locus (ASPL) at 17q25. It results in the formation of a fusion gene called ASPLTFE3 which in turn forms an aberrant protein giving it a TFE3 nuclear positivity on immunohistochemistry and responsible for its pathological manifestations. The messenger RNA expression of ASPL-TFE3 can be detected with the help of reverse transcriptionpolymerase chain reaction which provides a dependable molecular criterion for the confirmatory diagnosis of ASPS. ${ }^{8}$

On MRI, ASPS is seen as hyperintense T1weighted and T2-weighted images. Avid enhancement with contrast is also typical, with or without a nonenhancing necrotic core. On computed tomography (CT) images, large vessels and central nonenhancement indicating necrosis are a prominent feature of the tumor.

As ASPS is a known for its rich vascularity, radical resection is performed rather than fractional resection 
during surgery. Complete removal of the primary tumor prolongs patient survival time; however, it is still unclear whether postoperative radiotherapy provides any survival benefit. It has also been found to be insensitive to chemotherapeutic drugs, therefore no specific chemotherapeutic treatment regimens had been developed till now. However, the recent identification of the role of ASPSCR1- TFE3 in the MET signaling pathway promoting tumor cell proliferation and angiogenesis has assumed a vital role in the development of targeted molecular therapy in ASPS, namely antiangiogenic drugs and MET kinase inhibitors, with numerous ongoing clinical trials showing reassuring initial outcomes. ${ }^{9,10}$

\section{Conclusion}

ASPS is a rare soft tissue tumor that tends to recur or metastasize late in the follow-up period. Brain metastases should be considered in the differential diagnosis of an intracranial mass with the radiographic characteristics of a space occupying lesion, especially when the clinical or radiographic findings are even marginally unusual. TFE3 immunohistochemistry, special staining and knowledge of the characteristic microscopic features of ASPS facilitate an early diagnosis, with an early total resection possibly the most effective treatment for brain metastatic ASPS. To achieve extended disease-free survival, a multidisciplinary approach including surgery, radiotherapy and targeted chemotherapy must be utilized.

\section{References}

1. Ogose A, Morita T, Hotta T, Kobayashi H, Otsuka H, Hirata Y, Yoshida S. Brain metastases in musculoskeletal sarcomas. Jpn J Clin Oncol 1999;29:245-47.

2. Ahn SH, Lee JY, Wang KC, Park SH, Cheon JE, Phi JH, Kim SK. Primary alveolar soft part sarcoma arising from the cerebellopontine angle. Childs Nerv Syst 2014;30:345-50

3. Fox BD, Patel A, Suki D, Rao G. Surgical management of metastatic sarcoma to the brain. $J$ Neurosurg 2009;110:181-86.

4. Tao X, Hou Z, Wu Z, Hao S, Liu B. Brain metastatic alveolar soft-part sarcoma: Clinicopathological profiles, management and outcomes. Oncol Letters 2017;14(5):5779-84.

5. Christopherson WM, Foote Fw, Jr, Stewart FW. Alveolar soft-part sarcomas; structurally characteristic tumors of uncertain histogenesis. Cancer 1952;5:100-11.

6. Folpe AL, Deyrup AT. Alveolar soft-part sarcoma: A review and update. J Clin Pathol 2006;59:1127-32.

7. Lieberman PH, Brennan MF, Kimmel M, Erlandson RA, Garin-Chesa P, Flehinger BY. Alveolar soft-part sarcoma. A clinico-pathologic study of half a century. Cancer 1989;63:1-13.

8. Stacchiotti S, Negri T, Zaffaroni N, Palassini E, Morosi C, Brich S, Conca E, Bozzi F, Cassinelli G, Gronchi A, et al. Sunitinib in advanced alveolar soft part sarcoma: Evidence of a direct antitumor effect. Ann Oncol 2011;22:1682-90.
9. Tsuda M, Davis IJ, Argani P, et al. TFE3 fusions activate MET signaling by transcriptional up-regulation, defining another class of tumors as candidates for therapeutic MET inhibition. Cancer Res 2007;67(3):919-29.

10. Lazar AJ, Lahat G, Myers SE, et al. Validation of potential therapeutic targets in alveolar soft part sarcoma: an immunohistochemical study utilizing tissue microarray. 2009;55(6):750-55. 